UCB-PTH-04/21

LBNL-56050

SLAC-PUB-10580

SU-ITP-04/31

hep-th/0408085

\title{
Relativistic D-brane Scattering is Extremely Inelastic
}

\author{
Liam McAllister ${ }^{1}$ and Indrajit Mitra ${ }^{2,3}$ \\ ${ }^{1}$ SLAC and Department of Physics, Stanford University \\ Stanford, CA 94309/94305 \\ ${ }^{2}$ Berkeley Center for Theoretical Physics and Department of Physics \\ University of California, Berkeley, CA 94720-7300 \\ ${ }^{3}$ Theoretical Physics Group, Lawrence Berkeley National Laboratory \\ Berkeley, CA 94720-8162
}

\begin{abstract}
We study the effects of quantum production of open strings on the relativistic scattering of D-branes. We find strong corrections to the brane trajectory from copious production of highly-excited open strings, whose typical oscillator level is proportional to the square of the rapidity. In the corrected trajectory, the branes rapidly coincide and remain trapped in a configuration with enhanced symmetry. This is a purely stringy effect which makes relativistic brane collisions exceptionally inelastic. We observe that pair-creation of open strings could play an important role in cosmological scenarios in which branes approach each other at very high speeds.
\end{abstract}

August 2004 


\section{Introduction}

Thought experiments involving the scattering of strings or of D-branes provide the key to understanding certain essential phenomena in string theory. The discovery of strings in the theory is perhaps the most striking case, but other examples include the elucidation of the sizes of strings under various conditions and the appreciation of another length-scale in the dynamics of slow-moving D-branes.

Despite much early interest in the scattering of D-branes, certain important aspects of the dynamics have remained unexplored. In particular, the simplest treatments involve parameter regimes governed either by supergravity or by the effective worldvolume field theory of massless open strings. In the latter case, there can be significant quantum corrections arising from loops of light open strings or from pair-production of on-shell open strings.

A key consequence of the pair-production of open strings is the trapping of D-branes [1, which we now briefly review. Consider two Dp-branes, $p>0$, moving with a small relative velocity. As the branes pass each other, the masses of stretched open strings vary with time. This leads to pair production, in a direct analogue of the Schwinger pair-creation process for charged particles [2] or strings [3] in an electric field. Because the velocities are low, the production of stretched strings with oscillator excitations is highly suppressed. The resulting unexcited stretched strings introduce an energy cost for the branes to separate; unless these strings can rapidly annihilate, the branes will be drawn close together. In collisions with a nonzero impact parameter, the brane pair carries angular momentum; in this case the branes spiral around their center of mass, radiating closed strings, until eventually they fall on top of each other. The final outcome is that the open strings trap the branes in a configuration with enhanced symmetry. Because this process involves the production of only unexcited open strings, it falls within the purview of effective field theory.

Our goal is to explore related processes which are not describable in the low-energy effective field theory but which instead involve intrinsically stringy physics. We will show that the ultrarelativistic scattering of D-branes is a suitable laboratory for such an investigation, as corrections from the massive string states turn out to be essential. In particular, we will demonstrate that production of highly-excited open strings generates crucial corrections to the brane dynamics and leads to spectacular trapping of the branes over distances which can be of order the string length. As we will show, these corrections are much stronger than a naive application of effective field theory would predict; hence this is a setting where the importance of purely stringy effects is a surprise.

The intuition underlying this result is that in relativistic D-brane scattering, it should be possible to pair-produce highly-excited open strings. The density of string states at 
high excitation levels grows exponentially with energy; this is the well-known Hagedorn density of states. For this reason, even if the production of a given excited string state is exponentially suppressed compared to production of a massless string state, the competition of the growing and decaying exponentials will typically cause highlyexcited strings to dominate the process, in terms of both their number and their share of the total energy. Thus, one expects pair production of a huge number of highly excited strings. This is indeed the case, as was first explained by Bachas in the important work [4. Our further observation is that because the energy transferred into these open strings can easily be comparable to the initial kinetic energy of the D-brane pair, the massive open strings are absolutely central to the dynamics. This means that the backreaction arising from purely stringy effects is crucial.

We will study the effect on the dynamics of this explosive pair-production of massive modes. Our conclusion is that for a large range of velocities and impact parameters, almost all the initial kinetic energy of the branes is transferred to open strings and to closed string radiation. After the collision the branes are drawn together and come to rest. In near-miss scattering events with an impact parameter $b$, the branes revolve around their center of mass in a roughly circular orbit whose initial radius is of order $b$; this orbit swiftly decays via radiation of closed strings. This is to be contrasted to the much weaker trapping of nonrelativistic branes, which typically proceeds via very elliptical orbits, i.e. the stopping length is much greater than the impact parameter.

To recap, the dynamics of ultrarelativistic D-branes is strikingly inelastic: copious production of highly-excited stretched open strings rapidly drains the brane kinetic energy and traps the branes into a tight orbit, eventually leading the branes to coincide.

In this simple and controllable example it proves possible to understand aspects of the backreaction of open string production on the dynamics of colliding D-branes. The lessons of our analysis could be extended to cosmological models in which other sorts of fast-moving branes approach each other and collide. As we will discuss, these include the ekpyrotic/cyclic universe scenario, brane-antibrane scenarios, and the DBI model.

It is useful to indicate the various regions of parameter space that we will probe. We will outline this now to apprise the reader of our strategy; later, in $\S 5.3$, we will provide a more complete discussion.

The dimensionless quantities of interest are the impact parameter $b$ measured in units of the string length; the string coupling $g_{s}$, which determines the mass of the D-branes in string units; and the initial relative velocity of the branes $v$. We will find it more convenient to convert this velocity into the rapidity, $\eta \equiv \operatorname{arctanh}(v)$. We will usually set $\alpha^{\prime}=1$, except for a few cases where we will retain explicit factors of the string length for clarity.

Our goal in this work is to understand open string effects in relativistic dynamics; the nonrelativistic case is already well-understood [5, 1]. We will therefore impose 
$1-v \ll 1$ so that $\eta \gg 1$. Another important consideration is that the D-branes should have Compton wavelengths small compared to the impact parameter. Because the D-branes grow light at strong string coupling, this amounts to a requirement that the coupling should be sufficiently weak. Another obvious advantage of weak coupling is the suppression of string loop effects; our primary computation is a one-loop open string process. A further requirement is that the D-brane Schwarzschild radius should be much smaller than the impact parameter. This too can be achieved with a suitably small string coupling, as we will demonstrate in $\$ 5.3$. Furthermore, although energy loss through closed string radiation can be an important effect in a system of moving branes, there is a wide range of string coupling, depending on $\eta$, for which this effect is subleading compared to open string production. Although all these considerations show that weak coupling is desirable for control, it is important to recognize that as the coupling decreases, the D-branes grow heavy and hence stretch the strings farther before coming to rest.

In summary, there is a range of values of the string coupling in which the backreaction of open strings is significant and competing effects are suppressed.

The organization of this paper is as follows. First, in $\S 2$, we review the trapping of nonrelativistic branes, which provides the basic intuition for the more complicated, stringy process which we aim to study. Then, in $\S 3$, we study the interaction amplitude for moving branes. We compute the brane interaction via an annulus diagram and examine its imaginary part, which corresponds to open string pair production. This result is well-known, but we include it for logical completeness and to set our notation. Our primary result appears in $\S 4$, where we study the backreaction of open string production on the brane trajectory and estimate the stopping length on energetic grounds. In $\S 5$ we discuss potential corrections and additional effects, in particular the production of closed strings, and explain how they affect our considerations. We conclude with a few comments in $\S 6$. Finally, we collect useful identities about the theta functions in the appendix.

\section{Overview of the Trapping of Nonrelativistic Branes}

We will now briefly review the trapping of D-branes in nonrelativistic motion, which was studied in [1]. (See also [6, 7] for earlier work on related mechanisms in field theory and cosmology.) This process is governed by pair production of massless open strings and hence is describable in effective field theory. It provides the basic framework for understanding corrections to the brane dynamics, and so is a useful background for the stringy trapping which we will study in $§ 3$.

Because the field theory description is entirely sufficient, we can abstract the relevant 
properties of the worldvolume gauge theory and represent the system with a simplified model,

$$
\mathcal{L}=\frac{1}{2} \partial_{\mu} \phi \partial^{\mu} \bar{\phi}+\frac{1}{2} \partial_{\mu} \chi \partial^{\mu} \chi-\frac{g^{2}}{2}|\phi|^{2} \chi^{2}
$$

in which a complex scalar field $\phi$ couples to a real scalar field $\chi$. At the origin $\phi=0$, $\chi$ becomes massless.

Let us consider the trajectory

$$
\phi(t)=i \mu+v t
$$

in which $\phi$ is separated from the origin by the impact parameter $\mu$. This is a solution to the classical equations of motion of (11) provided that $\chi=0$. Along this trajectory, the mass of $\chi$ changes: in the limit where we impose (2) and ignore the effect of the coupling to $\chi$, we may rewrite (11) as

$$
\mathcal{L}=\frac{1}{2} \partial_{\mu} \phi \partial^{\mu} \bar{\phi}+\frac{1}{2} \partial_{\mu} \chi \partial^{\mu} \chi-\frac{g^{2}}{2}\left(\mu^{2}+v^{2} t^{2}\right) \chi^{2}
$$

so that the effective mass of $\chi$ varies with time. This results in production of $\chi$ quanta.

This effect is easily understood in the quantum mechanics example of a harmonic oscillator whose frequency changes over time from $\omega_{i}$ to $\omega_{f}$. If the oscillator begins in its ground state at frequency $\omega_{i}$ but the frequency changes nonadiabatically then the final state will not be the ground state of an oscillator of frequency $\omega_{f}$.

One can readily compute the occupation numbers $n_{k}$ of modes with momentum $k$. The result [1] is

$$
n_{k}=\exp \left(-\pi \frac{k^{2}+g^{2} \mu^{2}}{g v}\right) .
$$

If instead we consider a model in which the mass of $\chi$ is nowhere zero,

$$
\mathcal{L}=\frac{1}{2} \partial_{\mu} \phi \partial^{\mu} \bar{\phi}+\frac{1}{2} \partial_{\mu} \chi \partial^{\mu} \chi-\left(m^{2}+\frac{g^{2}}{2}|\phi|^{2}\right) \chi^{2}
$$

the result is instead

$$
n_{k}=\exp \left(-\pi \frac{k^{2}+m^{2}+g^{2} \mu^{2}}{g v}\right) .
$$

The crucial, though intuitive, observation is that production of a massive species is exponetially suppressed. For this reason, production of massive string modes is entirely negligible when the velocity is small.

We may now apply the result of the simplified model to a pair of D-branes. Suppose that two Dp-branes, $p>0$, are arranged to pass near each other. The brane motion changes the masses of stretched string states and induces pair production of unexcited 
stretched strings. As the branes begin to separate, these strings stretch and pull the branes back together.

This process can be followed in detail by numerically integrating the quantumcorrected equations of motion which follow from (11). Such an analysis was presented in [1]. However, analytical estimates are more readily generalized to the case of interest in this paper, which is the stringy scattering of relativistic branes. We will therefore explain how one can use energetics to estimate the stopping length in the system (1). (It was shown in [1] that such estimates are in excellent agreement with the numerical results, although only the nonrelativistic case was studied there.)

After the branes have passed each other, the stretched open strings grow in mass. Even though pair production has ceased, the energy contained in open strings grows with time, because the strings are being stretched:

$$
\rho_{\text {open }} \approx|\phi(t)| n_{\text {open }}
$$

When the energy in open strings is of the same order as the initial brane kinetic energy, the backreaction of the open strings is of order one and the brane motion slows down significantly. We therefore define the 'stopping length' $\phi_{*}$ via $\rho_{\text {open }}\left(\phi_{*}\right) \approx \frac{1}{2} T_{p} v^{2}$ where $T_{p}$ is the tension of a Dp-brane.

A few qualitative features of low-velocity trapping are worth mentioning. First, the greater the number density of produced strings, the shorter the stopping length. On the other hand, the stopping length increases if the brane velocity increases or the string coupling decreases (making the branes heavier in string units).

The behavior in the limit $v \rightarrow 1$ is not obvious a priori. To estimate the total number density $\nu_{\text {total }}$ of all string modes, we could take the nonrelativistic result (6) for the occupation numbers of a massive species and sum it over the levels $n$ in the string spectrum, including a factor of the density of states $N(n)$. The result (which was also presented in [1]) is

$$
\nu_{\text {total }} \propto \sum_{n=0}^{\infty} N(n) \exp \left(-\frac{2 \pi^{2}}{v}\left(n+\frac{b^{2}}{4 \pi^{2}}\right)\right) .
$$

As we explain in $\S 4.1$, the density of states at high levels $n$ obeys

$$
N(n) \sim n^{-11 / 4} \exp \left(\sqrt{8 \pi^{2} n}\right)
$$

This does not grow rapidly enough to compete with the exponential suppression (6) of high levels, so the limit $v \rightarrow 1$ does not display strong production of excited strings.

However, we will show in detail in $\S 3$, following [4, that the actual number density of produced strings is very much larger than the nonrelativistic estimate (8) suggests. We will find instead

$$
\nu_{\text {total }} \propto \sum_{n=0}^{\infty} N(n) \exp \left(-\frac{2 \pi^{2}}{\eta}\left(n+\frac{b^{2}}{4 \pi^{2}}\right)\right)
$$


where $\eta=\operatorname{arctanh}(v) \gg 1$. This result does not follow from special relativity alone; it is instead a stringy effect arising from velocity-dependent corrections to the stretched string masses, as we will show.

\section{The Interaction Amplitude for Moving D-branes}

We will now derive the interaction potential for two D-branes in relative motion with arbitrary velocity. Although this result is well-known 4, we include the calculation for completeness and to set notation.

\subsection{Interaction Potential from the Annulus Diagram}

We will derive the interaction potential by computing the open string one-loop vacuum energy diagram. This diagram is an annulus whose two boundaries correspond to the two D-branes. By the optical theorem, twice the imaginary part of this amplitude is the rate of pair production of on-shell open strings. Thus, our goal is to determine the imaginary part of the vacuum energy.

Several equivalent methods can be used to compute the vacuum energy. The original treatment 4 involves a direct computation of the spectrum of open strings between the moving branes; that is, it is possible to impose appropriate boundary conditions and solve for the mode expansion. The vacuum energy is then the sum of the zero-point energies of these oscillators.

We choose instead to review the perhaps more transparent computation given in [8]. Let us stress that in this subsection we follow the treatment of [8] in detail, with very minor modifications.

By double Wick rotation, a pair of branes in relative motion, separated by a transverse distance $b$, can be mapped to a stationary pair of branes at an imaginary relative angle, again separated by a distance $b$. We will make this precise below. Because the partition function for branes at angles is very well understood, the vacuum energy is easily computed in this approach.

Following [8], we begin with two D4-branes which are parallel to each other, extended along the directions $0,1,3,5,7$, and separated by a distance $b$ (the impact parameter) along $X^{9}$. (To regulate the computation we compactify the spatial dimensions on a $T^{9}$ of radius $R$.) Now let one brane move towards the other along the direction $X^{8}$ with velocity $v$. That is, the coordinates of the moving brane are $X^{8}=v X^{0}, X^{9}=b$ while the other brane has $X^{8}=X^{9}=0$. This is our actual problem.

We now perform the Wick rotation $X^{0} \rightarrow-i X^{\prime 7}, X^{7} \rightarrow i X^{\prime 0}$. This transforms the moving branes into static branes which are misaligned by an angle $\phi$ in the $\left(7^{\prime}, 8\right)$ 
plane. The angle $\phi$ is given by $X^{17} \tan \phi=X^{8}$. The brane velocity $v$ and rapidity $\eta$ are related to this angle by $\phi=-i \operatorname{arctanh}(v) \equiv-i \eta$.

Next, it is useful to combine the coordinates into complex pairs $Y_{a}$, where $Y_{1}=$ $X^{1}+i X^{2}, Y_{2}=X^{3}+i X^{4}, Y_{3}=X^{5}+i X^{6}, Y_{4}=X^{17}+i X^{8}$. Define also the angles $\phi_{1}=\phi_{2}=\phi_{3}=0, \phi_{4}=\phi$. The rotation then takes $Y_{4} \rightarrow \exp (i \phi) Y_{4}$. It is now a simple matter to set up the boundary conditions satisfied by strings which stretch between the branes:

$$
\begin{array}{ll}
\sigma_{1}=0: & \partial_{1} \operatorname{Re}\left[Y_{a}\right]=\operatorname{Im}\left[Y_{a}\right]=0 \\
\sigma_{1}=\pi: & \partial_{1} \operatorname{Re}\left[\exp \left(i \phi_{a}\right) Y_{a}\right]=\operatorname{Im}\left[\exp \left(i \phi_{a}\right) Y_{a}\right]=0 .
\end{array}
$$

The solutions to the wave equation which satisfy these boundary conditions are:

$Y_{a}(w, \bar{w})=\exp \left(-2 i \phi_{a}\right)\left[i \sqrt{\frac{\alpha^{\prime}}{2}} \sum_{r=\mathbf{Z}+\phi_{a} / \pi} \frac{\alpha_{r}^{a}}{r} \exp (i r w)\right]+\left[-i \sqrt{\frac{\alpha^{\prime}}{2}} \sum_{r=\mathbf{Z}+\phi_{a} / \pi} \frac{\alpha_{r}^{a *}}{r} \exp (i r \bar{w})\right]$,

where $w=\sigma_{1}+i \sigma_{2}$. We can readily write down the partition function for these four scalars:

$$
Z_{\text {scalar }}\left(\phi_{a}\right)=-i \frac{\exp \left(\phi_{a}^{2} t / \pi\right) \eta(i t)}{\theta_{11}\left(i \phi_{a} t / \pi, i t\right)}
$$

so that the resulting bosonic partition function is

$$
Z_{\text {boson }}=\prod_{a=1}^{4} Z_{\text {scalar }}\left(\phi_{a}\right)
$$

In a similar way, one can compute the fermionic partition function, keeping in mind the various spin structures:

$$
Z_{\text {ferm }}=\prod_{a=1}^{4} Z_{1}^{1}\left(\phi_{a} / 2, i t\right)
$$

where

$$
Z_{1}^{1}\left(\phi_{a} / 2, i t\right) \equiv \frac{\theta_{11}\left(i \phi_{a} t / 2 \pi, i t\right)}{\exp \left(\phi_{a}^{2} t / 4 \pi\right) \eta(i t)}
$$

We conclude that the one-loop potential is

$$
V=-\int_{0}^{\infty} \frac{d t}{t} \frac{1}{\sqrt{8 \pi^{2} \alpha^{\prime} t}} \exp \left(-\frac{t b^{2}}{2 \pi \alpha^{\prime}}\right) \prod_{a=1}^{4} \frac{\theta_{11}\left(i \phi_{a} t / 2 \pi, i t\right)}{\theta_{11}\left(i \phi_{a} t / \pi, i t\right)} .
$$

This potential governs D4-branes at a relative angle. To map into the case of interest, we T-dualize as many times as needed, each time introducing the replacement $\theta_{11}\left(i \phi_{a} t / \pi, i t\right) \rightarrow i \sqrt{8 \pi^{2} \alpha^{\prime} t} \eta^{3}(i t) / R$, where $R$ is the size of the spatial torus. 
This finally brings us to the potential for p-branes at an angle $\phi$ :

$$
V=-i R^{p} \int_{0}^{\infty} \frac{d t}{t}\left(8 \pi^{2} \alpha^{\prime} t\right)^{-p / 2} \exp \left(-\frac{t b^{2}}{2 \pi \alpha^{\prime}}\right) \frac{\theta_{11}(i \phi t / 2 \pi, i t)^{4}}{\theta_{11}(i \phi t / \pi, i t) \eta^{9}(i t)} .
$$

Our final interest is in the number density and energy density of open strings, so the spatial volume $R^{p} \equiv V_{p}$ will eventually cancel.

To read off the desired result for moving branes, we set $\phi=-i \eta$ to get

$$
V=-i V_{p} \int_{0}^{\infty} \frac{d t}{t}\left(8 \pi^{2} \alpha^{\prime} t\right)^{-p / 2} \exp \left(-\frac{t b^{2}}{2 \pi \alpha^{\prime}}\right) \frac{\theta_{11}(\eta t / 2 \pi, i t)^{4}}{\theta_{11}(\eta t / \pi, i t) \eta(i t)^{9}} .
$$

One can easily show that this agrees precisely with the result of [4], equation (11). To see this, use (71) and (77), define $t_{\text {there }}=2 t, \epsilon=\frac{\eta}{\pi}$, and set $\alpha^{\prime}=\frac{1}{2}$.

A useful equivalent form for (19) is

$V=-i V_{p} \int_{-\infty}^{\infty} d \tau \int_{0}^{\infty} \frac{d t}{t}\left(8 \pi^{2} \alpha^{\prime} t\right)^{-p / 2} \frac{\theta_{11}(\eta t / 2 \pi, i t)^{4}}{\theta_{11}(\eta t / \pi, i t) \eta(i t)^{9}} \exp \left(-\frac{t}{2 \pi \alpha^{\prime}}\left(b^{2}+v^{2} \tau^{2}\right)\right) \times \frac{v}{\pi} \sqrt{\frac{t}{2 \alpha^{\prime}}}$

In this form the time-dependence of the stretched string masses is manifest.

\subsection{Imaginary Part and Pair-Production Rate}

The above expression from the interaction potential is rich in information. The real part tells us about the velocity-dependent forces from closed string exchange, while twice the imaginary part is equal to the rate of production of open strings.

The potential (19) would be real if the integrand had no poles. However, $\theta_{11}(\eta t / \pi$, it) has a zero for integral values of $\eta t / \pi \equiv k$, so we can compute the imaginary part of the integral by summing the residues at the corresponding poles.

$$
\operatorname{Im}[V]=\frac{V_{p}}{2(2 \pi)^{p}} \sum_{k=1}^{\infty} \frac{1}{k}\left(\frac{\eta}{\pi k}\right)^{p / 2} \exp \left(-\frac{b^{2} k}{2 \eta}\right) Z(i k \pi / \eta)\left(1-(-1)^{k}\right),
$$

where we have defined the partition function $Z(\tau) \equiv \frac{1}{2} \theta_{10}^{4}(0 \mid \tau) \eta(\tau)^{-12}$. (The factor projecting out even values of $k$ arises because of Jacobi's 'abstruse identity'.)

This expression, which was first derived in [4, will be essential to our investigation. By extracting its behavior in various limits we will be able to study the effect of open string production on the brane dynamics.

First of all, we can check the normalization of (21) by taking the low-velocity limit, in which $\eta \rightarrow v$. The result is

$$
\operatorname{Im}[V]=\frac{8 V_{p}}{(2 \pi)^{p}} \sum_{k=1,3,5, \ldots}^{\infty} \frac{1}{k}\left(\frac{v}{\pi k}\right)^{p / 2} \exp \left(-\frac{b^{2} k}{2 v}\right) .
$$


This is identical to Schwinger's classic result [2] for the pair-production rate of electrons in a constant electric field. In the present case, the interpretation is of pair production of massless open strings between the branes, which was also obtained by the method of Bogoliubov coefficients in [1].

Our interest is in the case of velocities approaching the speed of light. We expect that the dominant contribution to pair production in this limit will come from highlyexcited string states. Because the density of states grows exponentially (9) at high levels, we anticipate copious production of massive strings and, as a result, dramatic backreaction on the brane motion.

To investigate this, we begin with the high-velocity limit $\eta \gg 1$ of (21):

$$
\operatorname{Im}[V]=\frac{V_{p}}{2(2 \pi)^{p}} \sum_{k=1,3,5, \ldots}^{\infty} \frac{1}{k}\left(\frac{\eta}{\pi k}\right)^{p / 2-4} \times \exp \left(\frac{\eta}{k}-\frac{b^{2} k}{2 \eta}\right)\left(1+\mathcal{O}\left(e^{-\eta / k}\right)\right)
$$

where we have used the asymptotics (74).

Keeping the dominant contribution, which comes from $k=1$, and expressing the result as a number density $\nu_{\text {open }}$ of open strings stretching between the branes, we find

$$
\nu_{\text {open }} \approx c_{p} \eta^{\frac{p}{2}-4} \times \exp \left(\eta-\frac{b^{2}}{2 \eta}\right)
$$

where $c_{p}=\left(2(2 \pi)^{p} \pi^{p / 2-4}\right)^{-1}$.

There are three important differences between the low-velocity effect in [2] and the high-velocity relation of (24) . The first is that production of strings is exponentially suppressed at low velocities: this can be understood from the fact that the amount of strings produced at a given energy falls off exponentially with energy, while the density of states for such low energies is a simple power law. At high energies, however, the density of states grows exponentially and these two competing exponentials lead to copious string production if the initial velocity of the branes is sufficiently high.

The second important difference is that at low velocities, the efficacy of the trapping process is strongly dependent on the impact parameter. For large impact parameters, $b \gg 1$, (recall that $b$ is measured in string units) the trapping is exponentially weak. For ultrarelativistic branes, however, the trapping weakens only when $b \gg \eta$. The effective range of strong trapping is evidently much increased in the ultrarelativistic limit.

Finally, in the low-velocity limit, the energy of produced open strings is a negligible fraction of the D-brane energy [1] until the branes separate far enough to stretch the open strings significantly. The associated distance, the 'stopping length', is generically much larger than the impact parameter. In the ultrarelativistic limit, in contrast, the energy carried by the open strings can be comparable to the brane kinetic energy even 
before any stretching. This occurs because high speeds make possible the production of highly-excited strings with significant oscillator energy. This consideration suggests that the backreaction of open strings is much more dramatic for relativistic branes than for nonrelativistic ones. We undertake a careful study of this in the following section.

\section{$4 \quad$ Backreaction from Energetics}

We have seen in the previous section that relativistic brane motion leads to the production of a tremendous number density (24) of stretched open strings. We would now like to estimate the effect of this process on the brane motion, and to do so we must estimate the energy density carried by the produced open strings.

\subsection{Open String Energy}

This energy is easily computed if we first rewrite the partition function $Z$ as a sum over string states. This is conveniently parametrized in terms of the excitation level $n$ and the number of states $N(n)$ at each level.

$$
Z(i k \pi / \eta) \equiv \frac{1}{2} \theta_{10}(0, i k \pi / \eta)^{4} \eta(i k \pi / \eta)^{-12}=\sum_{n=0}^{\infty} N(n) \exp \left(-\frac{2 \pi^{2} n k}{\eta}\right) .
$$

We would first like to determine the behavior of $N(n)$ at high excitation levels $n$. Taking the ansatz

$$
N(n) \approx c_{N} n^{a} \exp (b \sqrt{n})
$$

approximating the sum by an integral, evaluating this integral by stationary phase, and demanding the asymptotics (174), we find

$$
N(n) \approx(2 n)^{-11 / 4} \exp (\pi \sqrt{8 n}) .
$$

The numerical prefactor was chosen for convenience; strictly speaking, the approximate

evaluation of the integral does not determine constant prefactors of order unity, but for our purposes it suffices to choose the factor now as in (27).

With this result in hand, we can rewrite (21) as

$$
\operatorname{Im}[V]=\frac{V_{p}}{(2 \pi)^{p}} \sum_{k=1,3, \ldots}^{\infty} \frac{1}{k}\left(\frac{\eta}{\pi k}\right)^{p / 2} \exp \left(-\frac{b^{2} k}{2 \eta}\right) \sum_{n=0}^{\infty} N(n) \exp \left(-\frac{2 \pi^{2} n k}{\eta}\right) .
$$

An equivalent form for this relation is

$$
\operatorname{Im}[V]=\frac{\sqrt{2} v V_{p}}{(2 \pi)^{p+1}} \int_{-\infty}^{\infty} d \tau \sum_{k=1,3, \ldots}^{\infty} \frac{1}{k}\left(\frac{\eta}{\pi k}\right)^{\frac{p-1}{2}} \sum_{n=0}^{\infty} N(n) \exp \left(-\frac{2 \pi^{2} k M^{2}(\tau)}{\eta}\right) .
$$


where

$$
M^{2}(\tau) \equiv \frac{n}{\alpha^{\prime}}+\frac{b^{2}+v^{2} \tau^{2}}{4 \pi^{2} \alpha^{\prime 2}}
$$

To determine the total energy of the produced strings, we begin with the energy of a string at level $n$, when the separation of the branes along the direction of motion is $r$ :

$$
E(n)^{2}=\frac{v^{2}}{\eta^{2}}\left(\frac{n}{\alpha^{\prime}}+\frac{b^{2}+r^{2}}{4 \pi^{2} \alpha^{\prime 2}}\right)
$$

The velocity-dependence is perhaps counterintuitive, but can be derived by requiring consistency of the annulus result (21) with a steepest-descent computation in the nearly-adiabatic limit $b \gg \eta, \eta \gg 1$. However, we are not aware of a simple, a priori computation of this mass correction. Note that the mass (31) does reduce to the correct rest mass in the limit of small velocity.

We can now express the energy density of produced open strings as

$$
\rho_{\text {open }}=\frac{1}{(2 \pi)^{p}} \sum_{k=1,3, \ldots}^{\infty} \frac{1}{k}\left(\frac{\eta}{\pi k}\right)^{p / 2} \exp \left(-\frac{b^{2} k}{2 \eta}\right) \sum_{n=0}^{\infty} E(n) N(n) \exp \left(-\frac{2 \pi^{2} n k}{\eta}\right) .
$$

Because of the competition of the growing and decaying exponential factors, this sum is dominated by terms near some $n_{\text {peak }} \gg 1$. As indicated above, we approximate the sum on levels using the relation

$$
\sum_{n=0}^{\infty} N(n) n^{\alpha} \exp \left(-\frac{2 \pi^{2} n}{\eta}\right) \approx 2^{-11 / 4} \int_{n_{0}}^{\infty} d n n^{\alpha-11 / 4} \exp \left(\pi \sqrt{8 n}-\frac{2 \pi^{2} n}{\eta}\right)
$$

where the lower bound $n_{0}>0$ is chosen so that the integral is dominated by $n \approx n_{\text {peak }}$, not $n \approx 0$. We have kept the leading term in the sum on $k$. By the method of stationary phase we find that the integral is dominated by $n \approx n_{\text {peak }}=\eta^{2}\left(2 \pi^{2}\right)^{-1}$, leading to

$$
2^{-11 / 4} \int_{n_{0}}^{\infty} d n n^{\alpha-11 / 4} \exp \left(\pi \sqrt{8 n}-\frac{2 \pi^{2} n}{\eta}\right) \approx \frac{1}{2} e^{\eta}\left(\frac{\pi}{\eta}\right)^{4}\left(\frac{\eta^{2}}{2 \pi^{2}}\right)^{\alpha} .
$$

For $\alpha=0$ this reproduces the asymptotic behavior (174); we normalized (27) to arrange this.

This approximate result provides an important physical lesson: the primary contribution to the open string energy comes from strings at levels $2 \pi^{2} n \approx \eta^{2}$. For such a string,

$$
E(n)=\frac{v}{2 \pi \eta} \sqrt{4 \pi^{2} n+b^{2}+r^{2}} \approx \frac{1}{2 \pi} \sqrt{2+\frac{b^{2}+r^{2}}{\eta^{2}}} .
$$

Let us now examine this result in the parameter ranges of interest. If the stretched string length is large compared to $\eta, \sqrt{b^{2}+r^{2}} \gg \eta$, then the sum (32) is simply

$$
\rho_{\text {open }} \approx \frac{\sqrt{b^{2}+r^{2}}}{2 \pi \eta} \nu_{\text {open }}
$$


On the other hand, when the rapidity is larger than the separation, $\eta \gg \sqrt{b^{2}+r^{2}}$, we have instead

$$
\rho_{\text {open }} \approx \frac{1}{\pi \sqrt{2}} \nu_{\text {open }}
$$

where we have used (34) with $\alpha=1 / 2$.

The key observation which follows from (37) is that the energy density carried by produced pairs of stretched open strings can be a significant fraction of the kinetic energy density of the Dp-brane. The backreaction from open string production is therefore an important contribution to the dynamics of relativistic D-branes. We will now examine this in detail.

\subsection{Estimate of the Stopping Length}

It will be very important to recognize three length-scales which arise in the problem: the effective size $r_{\text {eff }}(\eta)$ of a relativistic brane, the critical impact parameter $b_{\text {crit }}(\eta)$ beyond which the trapping rapidly weakens, and the size $r_{n a d}(\eta)$ of the region in which the stretched open string masses change nonadiabatically.

The factor depending on $b$ in (24) indicates that the effective area of a brane moving with rapidity $\eta$ is 4 ]

$$
r_{e f f}^{2} \approx \eta \alpha^{\prime}
$$

This corresponds precisely to the logarithmic growth in cross-sectional area of a highlyboosted fundamental string, $r_{\text {eff }}^{2} \sim \alpha^{\prime} \ln \left(\alpha^{\prime} s\right)$, where $\sqrt{s}$ is the center-of-mass energy. The explanation for this growth is that a Regge probe of an ultrarelativistic string is sensitive to rather high-frequency virtual strings, whose considerable length creates a large cloud of virtual strings 9]. We conclude that a D-brane with rapidity $\eta$ has an apparent radius $r_{\text {eff }}=\sqrt{\eta \alpha^{\prime}}$.

The growth in effective area provides an additional perspective on the velocitydependent mass (31) of stretched strings. Both effects may be considered to originate in a rescaling of the effective string tension,

$$
T(\eta)=\frac{1}{2 \pi \alpha^{\prime} \eta}
$$

This results in a D-brane size $r_{e f f} \approx \sqrt{\eta \alpha^{\prime}}$ and a stretched string mass

$$
m_{e f f}=\frac{1}{\sqrt{\eta \alpha^{\prime}}} \sqrt{n+\frac{b^{2}}{4 \pi^{2}}}
$$

consistent with (38), (31). This rescaling of the effective tension is a very useful heuristic for understanding the dramatic difference between the naive result (8) and the 
complete annulus computation (24) for the number density. This rescaling can also be understood from the T-dual electric field perspective: as the electric field approaches a critical value, the strings can no longer hold themselves together, so their effective tension goes to zero [3].

Next, to find the critical impact parameter, we note that the open string energy density obeys

$$
\rho_{\text {open }} \propto \exp \left(\eta-\frac{b^{2}}{2 \eta}\right),
$$

so that for $\eta \gg 1$, the critical distance is evidently $b_{\text {crit }} \sim \eta$. For impact parameters less than $b_{\text {crit }}$, the open string energy density is generically large. The trapping effect is therefore very strong for impact parameters of order $b_{\text {crit }}$ and smaller. (Nevertheless, trapping still occurs for impact parameters much larger than $b_{\text {crit }}$.)

Finally, the nonadiabaticity is characterized by how rapidly the frequency changes with time. Quantitatively, it is measured by the dimensionless quantity $\xi \equiv \frac{\dot{\omega}}{\omega^{2}}$, where $\omega$ is the frequency. Using (30) we find

$$
\xi=\frac{2 \pi \eta r \dot{r}}{\left(4 \pi^{2} n+b^{2}+r^{2}\right)^{3 / 2}} \approx \frac{2 \pi r \eta}{\left(2 \eta^{2}+b^{2}+r^{2}\right)^{3 / 2}}
$$

which reaches its peak at $r^{2}=\eta^{2}+\frac{1}{2} b^{2}$. Thus, the region of nonadiabaticity has size $r_{n a d} \sim \eta$. Open strings are produced in large quantities when $-r_{\text {nad }} \lesssim r \lesssim r_{n a d}$.

In summary, the critical impact parameter is $b_{\text {crit }} \sim \eta$, which is also the size of the nonadiabatic region. The effective radius of a moving D-brane, i.e. the size of the stringy halo, is much smaller, $r_{\text {eff }} \sim \sqrt{\eta} \ll b_{\text {crit }}$. For any fixed, large $\eta$ we can require

$$
r_{\text {eff }} \ll b \ll b_{\text {crit }}
$$

so that the trapping is very strong but the stringy halos are small enough to be unimportant. The case of a head-on collision, $b \lesssim r_{\text {eff }}$, is also interesting, particularly for the question of string production in the cyclic universe models, but we will first explore the better-controlled regime (43).

One further observation is that a scattering event with impact parameter $b$, no matter how powerful the trapping, will typically involve motion on an arc whose initial radius is at least of order $b$. Angular momentum conservation prevents the moving brane from coming abruptly to a complete stop; over one or more orbits, however, there is sufficient time to radiate away the angular momentum into closed string modes, as we will see in $\$ 5.1$. In strong trapping, such as we will find in the relativistic case, the orbits will be roughly circular, whereas in nonrelativistic trapping the typical orbit is very highly elliptical, indicating weaker binding.

With these estimates in hand we can at last compute the stopping length for a scattering event. Taking one brane to be at rest and the other to have velocity $v$, we 
define as before

$$
\eta \equiv \operatorname{arctanh}(v)
$$

Working instead in the center of mass frame, the branes approach each other with velocities

$$
u=\tanh (\omega)=\tanh (\eta / 2)
$$

so that the center-of-mass $\gamma$ factor for either brane is

$$
\gamma=\frac{1}{\sqrt{1-u^{2}}} \sim \frac{1}{2} e^{\omega}
$$

when $\omega \gg 1$. The energy density of the brane pair is then

$$
E_{t o t}=2 T_{D p} \gamma \sim T_{D p} e^{\omega}=T_{D p} e^{\eta / 2} .
$$

We therefore find that for $\eta \rightarrow \infty$,

$$
\rho_{D p}=T_{D p} e^{\eta / 2}=\frac{1}{g_{s}(2 \pi)^{p}} e^{\eta / 2}
$$

In the case of strong trapping, $b \ll b_{\text {crit }} \approx \eta$, the open string energy at the minimum brane separation is

$$
\rho_{\text {open }} \approx \frac{c_{p}}{\pi \sqrt{2}} \eta^{\frac{p}{2}-4} \exp (\eta)
$$

whereas for weak trapping, $b \gg b_{\text {crit }}$, the open string energy is instead

$$
\rho_{\text {open }} \approx \frac{c_{p}}{2 \pi} \eta^{\frac{p}{2}-5} \sqrt{b^{2}+r^{2}} \exp \left(\eta-\frac{b^{2}}{2 \eta}\right)
$$

where $c_{p}=\left(2(2 \pi)^{p} \pi^{p / 2-4}\right)^{-1}$. Of course, the open string energy depends on $r$ even in the case of strong trapping, but this dependence is relatively unimportant until $r \sim \eta$.

Comparing (48), (49) we conclude that if an external force compels the branes to pass each other at constant, ultrarelativistic velocity, then, unless the string coupling is exponentially small, the energy stored in open strings at the point of closest approach is considerably larger than the initial kinetic energy of the branes. This means that without an artificial external force, the branes will not pass each other with undiminished speed, as this is energetically inconsistent.

We expect instead that as open strings are produced, the branes slow down gradually, leading to diminished further production of strings. The final result, of course, will be consistent with conservation of energy. (In $\S 4.3$ we will address the production of open strings between decelerating branes, and in $§ 5.1$ we will explain that the emission of closed string radiation also serves to reduce the rate of production of open strings.) 
Although the open string energy in (49) is an overestimate for the reason just mentioned, we will nevertheless use it now to find an estimate of the stopping length. This will serve to illustrate our technique in a manageable setting; it will then be a simple matter to repeat the analysis including the corrections of $\S 4.3$ and $\S 5.1$, which will not alter the form of our result.

We define the stopping length $r_{*}$ by $\rho_{\text {open }}\left(r_{*}\right)=\rho_{D p}$, so that at $r=r_{*}$ all the initial energy has been stored in stretched open strings. Equating (48) and (50), we find the stopping length

$$
r_{*} \approx \frac{4 \pi^{2}}{g_{s}} \exp \left(-\frac{\eta}{2}+\frac{b^{2}}{2 \eta}\right)\left(\frac{\eta}{\pi}\right)^{5-p / 2} .
$$

This is our main result. It manifests the surprising property that for sufficiently large rapidity, the stopping length decreases as the rapidity increases. (More precisely, for any fixed $g_{s}, b$ there exists a rapidity $\eta_{\min }$ such that the stopping length decreases as $\eta$ increases past $\eta_{\min }$.) To understand this unusual property, it is useful to keep in mind the behavior of D-branes scattering at even greater speeds, so great that the stringy halos themselves collide. For any $b$ there is an $\eta$ such that $r_{e f f} \gtrsim b$; the scattering of the branes is then described by the collision of absorptive disks of radius $r_{\text {eff }}$ [4. Moreover, for a suitable range of $g_{s}$ the brane Schwarzschild radii are so large that black hole production is an important consideration. We have carefully chosen our parameter ranges to exclude these effects and focus instead on the more controllable regime of strong stringy trapping; however, the black disk collisions and black hole production serve to illustrate that the limit of arbitrarily high rapidity involves very hard scattering and high inelasticity, in good agreement with the large- $\eta$ behavior of (51).

The stopping length (51) is large in string units only when

$$
g_{s} \ll 4 \pi^{2} \exp \left(-\frac{\eta}{2}+\frac{b^{2}}{2 \eta}\right)\left(\frac{\eta}{\pi}\right)^{5-p / 2}
$$

which is an exponentially small value of the coupling provided $\eta \gg b, \eta \gg 1$. Thus, although backreaction from open string production is a higher-order correction to the dynamics [10] which one might suppose is unimportant at moderately weak coupling, we have shown that for relativistic branes with $b \ll \eta$ the backreaction of open strings is crucial unless the string coupling is extraordinarily small.

\subsection{Corrections from Deceleration}

All of our computations so far have applied exclusively to a pair of branes approaching each other at constant velocity. On the other hand, we have demonstrated that the 
backreaction from open string production, as computed along this trajectory, necessarily causes the branes to decelerate. Clearly, the next step is to understand how the amount of string production changes when the branes follow a decelerating trajectory.

The analysis of string production during deceleration turns out to be tractable in the nonrelativistic limit. However, we have not found an exact answer for the relativistic case. Upon double Wick rotation the amount of string production between decelerating branes is mapped to the interaction between curved branes, which is not obviously solvable with conformal field theory techniques.

Even though we will not find an exact result for the string production, we will be able to place bounds on the resulting number density. This is sufficient information for the analysis of $\S 4.3$ : although our results there for the stopping length will not be as precise as they are in the nonrelativistic limit, the qualitative features - copious production of excited strings, rapid trapping, and very high inelasticity - will be quite clear.

First, however, we will examine the limit of instantaneous deceleration. Take the branes to move with a velocity $v_{0}$ for all $t<0$, but to come to rest for $t>0$. This problem can be solved exactly by matching the parabolic cylinder functions (and their derivatives) to the plane wave solutions at $t=0$. However, this setup clearly involves enormous non-adiabaticity and so there would be an extremely large amount of pairproduction, far greater even than in the case of constant velocity. This is readily computed, but it is not useful; we would like a more conservative estimate.

A more realistic picture is one in which the relative velocity of the branes varies as a function of time, for example as $v(t)=v_{0}(1-\tanh (t / f))$, where $f$ measures how abruptly the brane slows down. (Note also that in this setup the initial velocity is $v(-\infty)=2 v_{0}$.) The wave equation governing the stretched strings is therefore

$$
\left(\partial_{t}^{2}+k^{2}+g^{2} b^{2}+g^{2} v_{0}^{2}[t-\log (\cosh (t / f))]^{2}\right) \chi=0 \text {. }
$$

It is instructive to consider the non-adiabaticity parameter $\xi \equiv \dot{\omega} / \omega^{2}$, where

$$
\omega^{2}(t)=k^{2}+g^{2} b^{2}+g^{2} v_{0}^{2}[t-\log (\cosh (t / f))]^{2} .
$$

Let us first take $f \ll 1$, which is the case of very rapid deceleration. In this limit the deceleration is concentrated at $t=0$, so that for slightly later times, when the branes have come to a halt, we have $\xi=0$ and hence no particle production. Comparing this scenario to that of branes moving with uniform velocity $2 v$ and no deceleration, we see that an abrupt stop reduces the effective time available for particle production by a factor of two. Thus, for branes which come to a halt very rapidly, the total number of particles produced is approximately half the number produced when the branes move with uniform velocity. 
We can analytically solve the problem in the opposite limit of very gentle deceleration, $f \gg \sqrt{k^{2}+g^{2} b^{2}} /\left(g v_{0}\right)$. Using the steepest descent method to determine the Bogoliubov coefficients [11, 12, 13] and observing that in this limit there is a branch point very near the imaginary axis, at $-i \sqrt{k^{2}+g^{2} b^{2}} /\left(g v_{0}\right)$, we find

$$
\left|\beta_{k}\right|^{2}=\exp \left(-\pi\left(k^{2}+g^{2} b^{2}\right) /\left(g v_{0}\right)\right)
$$

This coincides with the exact result for the constant-velocity problem with velocity $v(t)=v_{0}$. However, as we already noted, in the present case the initial velocity is $v(-\infty)=2 v_{0}$. Our very simple conclusion is that this gradually decelerating trajectory leads to the same amount of string production as an unaccelerated trajectory in which the branes move at a uniform velocity which is smaller by a factor of two. The effective velocity, for purposes of particle production, is thus the average velocity $\frac{1}{2}(v(-\infty)+$ $v(\infty))$.

We conclude that very gradual deceleration results in significantly reduced string production. In particular, comparing the limits of large and small $f$, we see that the reduction in number density is much greater for gradual than for rapid deceleration.

The above result applies to nonrelativistic motion. The string computation which would be analogous to the annulus partition function but incorporate deceleration is considerably more complicated. In particular, the acceleration of the branes breaks conformal invariance, so it is difficult to use conventional techniques to compute the string production in this case.

Fortunately, it is possible to estimate the stopping length without an exact result for the string production during deceleration. The simple argument relies only on energetics and on the constant-velocity result (24).

Suppose that open string production slows a moving brane, bringing it from an initial kinetic energy $E_{i}=\gamma_{i} T_{p}$ to an energy (at the point of closest approach) $E_{f}=\gamma_{f} T_{p}$, where $\gamma_{i}, \gamma_{f}$ are the usual relativistic factors. The stopping length, defined again by $E_{i}=E_{\text {open }}\left(r_{*}\right)$, is easily seen to be

$$
r_{*} \approx \frac{2 \pi \eta E_{i}}{\nu_{\text {open }}}=\sqrt{2} \eta \frac{E_{i}}{E_{\text {open }}}=\sqrt{2} \eta \frac{E_{i}}{E_{i}-E_{f}},
$$

where we have used (36), (37).

Consider first the case $\gamma_{f} \gg 1$. If the stopping length is large compared to the size $r_{n a d}$ of the nonadiabatic region, $r_{*} \gg \eta$, then the branes are moving quickly as they leave the region of nonadiabaticity. This means that the result (24) applies directly, and we return to an apparent inconsistency: the open string energy is large compared to the initial energy. This is a clear signal that the stopping length cannot be much larger than $r_{\text {nad }} \sim \eta$. 
A stopping length of order $\eta$ or smaller is indicative of strong trapping: the branes come to rest around the time that the nonadiabaticity grows small, which means that a few strings are still being produced. Furthermore, this distance $\eta$ is comparable to the critical impact parameter and critical orbital radius.

On the other hand, in the case $\gamma_{f} \sim 1$, we have $E_{f} \ll E_{i}$, so that (56) yields the stopping length $r_{*} \approx \sqrt{2} \eta$.

We conclude that no matter how the deceleration affects open string production, if the only process acting to slow the branes is loss of energy to open strings, then the stopping length is no more than of order $\eta$, i.e. the size of the nonadiabatic region. Thus, the trapping is very strong: very little stretching is required before the branes are brought to rest.

Given a good estimate of the open string production along a decelerating path, we could give a more accurate estimate of the stopping length. However, we have just demonstrated through energetics and the result (24) that in any event this stopping length is no larger than $\eta$. In fact, we expect that it is actually considerably smaller than this.

It remains a possibility that loss of energy through closed string radiation could modify this result. We now proceed to show that this is not the case.

\section{$5 \quad$ Further Considerations}

\subsection{Production of Closed Strings}

By incorporating the effects of open string production we have seen that relativistic D-branes decelerate abruptly as they pass each other. This deceleration will lead to radiation of closed strings, in a process analogous to bremsstrahlung. This drains energy from the brane motion, and, unlike the transfer of energy into stretched open strings, this energy is forever lost from the brane system. Closed string radiation therefore serves to increase the inelasticity of a brane collision. Now, the end state of a near-miss is a spinning 'remnant', i.e. two D-branes orbiting rapidly around each other, connected by a high density of strings. Loss of energy and angular momentum to closed string radiation will swiftly reduce the rotation of this remnant, at least until the velocities become nonrelativistic.

One potential worry is that the energy loss to radiation might be so large that the quantity of open strings produced during a near-miss is quite small, leading to weak trapping and a large stopping length. This is an example of the more general concern that string production could be highly suppressed if any other effect caused the branes to decelerate to nonrelativistic speeds before reaching each other. We will show that 
the radiation of massless closed strings can be energetically significant but, even so, does not alter our conclusion that the stopping length is not large in string units.

To estimate the energy emitted as massless closed strings, we will make use of the close analogy of this process to gravitational bremsstrahlung [15] and to gravitational synchrotron radiation [14]. Of course, one of the massless closed string modes is the graviton, but we also expect radiation of scalars, including the dilaton and, when present, the compactification moduli. Even so, it will not be at all difficult to convert results from general relativity to the case at hand, because in practice, relativists often use the far simpler scalar radiation to estimate the basic properties of gravitational radiation. We will do the same.

Consider a small mass $m$ moving rapidly past a large mass $M$ in a path which is, to first approximation, a straight line. A burst of gravitational radiation will be emitted in a very short time, at the moment of closest approach. This is called gravitational bremsstrahlung. The peak radiated power is approximately [15]

$$
P \sim \frac{G^{3} M^{2} m^{2}}{b^{4}} \gamma^{4}
$$

where $G$ is the Newton constant, $b$ is the impact parameter, and $\gamma$ is the relativistic factor. For the remainder of this section we omit numerical prefactors: it will suffice to have the dimensional factors and the powers of $\gamma$.

The case of interest to us is extremely strong binding by open strings, for if the acceleration caused by the open strings is small then the closed string radiation should not play a key role, and the argument for trapping given in $\S 4.3$ suffices. Thus, we model the brane scattering by a gravitational scattering event in which the impact parameter is not much larger than the Schwarzschild radius of the larger mass. This gives

$$
P \sim \frac{G m^{2}}{b^{2}} \gamma^{4}
$$

Another useful case is that of gravitational synchrotron radiation from a mass $m$ moving in a circular orbit with period $\omega_{0}$. The power is [14]

$$
P \sim G m^{2} \omega_{0}^{2} \gamma^{4} \sim \frac{G m^{2}}{b^{2}} \gamma^{4}
$$

where we have identified the inverse frequency with the minimum expected orbital radius, which is of order the impact parameter. This result will be very useful for understanding the decay of the initial circular orbit.

Furthermore, one can directly compute, in the supergravity limit, the radiation from an accelerated D-brane. The result for circular motion with radius $b$ is [16]

$$
P=\frac{G m^{2}}{b^{2}} \gamma^{4}
$$


The results (59), (58), and (60) are thus in good agreement.

Knowing now the power lost to closed strings for a given decelerating trajectory, we also wish to compute the quantity of open strings which would be required to produce this trajectory. Stated more generally, given an object being accelerated by an external force, we are interested in the ratio of the radiated power to the power associated with the driving force. For an accelerating electron this is a textbook problem; see e.g. [17], chapter 14 .

The result is that there is a characteristic length $L_{e}=\frac{2}{3} \frac{e^{2}}{m c^{2}}$ governing radiation by electrons, and unless an electron's energy changes by of order its rest energy during acceleration over a distance of order $L_{e}$, the radiation is negligible compared to the external power. More specifically,

$$
\frac{E_{\text {radiated }}}{E_{\text {driving }}} \equiv \Omega_{e} \approx \frac{\Delta E}{\Delta x} \frac{L_{e}}{m c^{2}}
$$

where the total change in energy, from all causes, is $\Delta E$ over a distance $\Delta x$.

One can readily estimate the corresponding characteristic length $L_{D}$ for massless closed string radiation from a D-brane by comparing to the power (60). The outcome is that $L_{D} \sim g_{s} l_{s}$.

Let us now consider a brane whose initial kinetic energy is $E_{i}=\gamma_{i} T_{p}$, where $\gamma_{i} \gg 1$. Suppose that the brane decelerates over a distance $\Delta x$ to a new kinetic energy $E_{f}=$ $\gamma_{f} T_{p}, \Delta \gamma \equiv \gamma_{i}-\gamma_{f}$. The 'driving force' here is loss of energy through open string production; we will now compare this to the energy lost to radiation.

$$
\Omega_{D} \equiv \frac{E_{\text {closed }}}{E_{\text {open }}} \approx \frac{\Delta E}{T_{p}} \frac{L_{D}}{\Delta x}=g_{s} \Delta \gamma \frac{l_{s}}{\Delta x}
$$

If $\Omega_{D} \ll 1$ then our previous conclusions hold automatically, as the closed strings are energetically negligible. If $\Omega_{D} \gg 1$, there are two cases to consider. First, if $\gamma_{f} \sim 1$, so that $\Delta \gamma \sim \gamma_{i} \gg 1$, the branes have slowed down to nonrelativistic motion. In this case the energy in open strings can be estimated to be

$$
E_{\text {open }} \approx \frac{\Delta E}{\Omega_{D}} \approx \frac{T_{p}}{g_{s}} \frac{\Delta x}{l_{s}}
$$

To arrive at this rough estimate we did not need the Bogoliubov coefficients derived from the annulus amplitude; we have used instead the fact that the external driving force (open string production) can be determined based on the postulated trajectory. Proceeding to estimate the stopping length, we find

$$
\frac{r_{*}}{l_{s}} \approx \frac{2 \pi \eta E_{f}}{\nu_{\text {open }}} \approx \frac{\sqrt{2} \eta E_{f}}{E_{\text {open }}} \ll \frac{\eta T_{p}}{E_{\text {open }}}=\eta g_{s} \frac{l_{s}}{\Delta x} .
$$


The distance $\Delta x$ is of order $\eta$, because that is the size of the nonadiabatic region in which open strings are created. To make a very conservative estimate, we will use $\Delta x \gtrsim l_{s}$. Then, because we are working at weak string coupling, the stopping length is

$$
\frac{r_{*}}{l_{s}} \ll \eta g_{s} \frac{l_{s}}{\Delta x} \ll \eta
$$

so that the stopping length is much smaller than $\eta l_{s}$.

The second case is $\Omega_{D} \gg 1, \gamma_{f} \gg 1$, so that the brane is moving relativistically even after decelerating, and the relative velocity is large when the branes pass each other. Our general conclusion will be invalid only if the branes do not rapidly trap in this final case. However, if the branes separate to a considerable distance while moving rapidly, our annulus amplitude computation of open string production applies directly. In other words, by assuming that the branes can separate, we are arranging that they leave the region of nonadiabaticity, so that the number density of open strings is accurately given by (24), and the trapping length by (51). Thus, the assumption that the branes separate at high speed is not consistent.

We conclude that closed string emission can slow the motion of the brane pair, but it does not substantially increase the stopping length. In fact, radiation helps considerably to bring the branes to rest: once the branes are trapped and are spiraling around each other, rapid radiation losses will slow their rotation. This is enhanced by the familiar fact that, for relativistic objects, radiation losses are greater in circular motion than in rectilinear accelerated motion. Once the branes are trapped they slow down through this closed string synchrotron radiation. From the power (59) we conclude that the branes lose energy so rapidly that they would require only a few orbits to come to rest. In practice the spin-down process is prohibitively complicated, but this result suffices to show that the lifetime of the highly-excited, rapidly revolving remnant is in any case very short.

One important additional point is that the closed string radiation is strictly negligible only when the coupling is so small that the branes are rather heavy, and hence stretch the open strings farther before stopping. There is consequently a tradeoff between computability and control, which are best at extremely weak coupling, and the strength of the trapping, which is best for couplings above the bound (52). It is essential to recognize that for any nonzero coupling, the collision is inelastic and trapping eventually does occur; however, the stopping length increases when the coupling grows very small.

A further question which we have not addressed is the production of massive closed strings. In the case of very abrupt deceleration we would expect nonvanishing production of these modes. We will leave a precise computation of this effect within string theory as an interesting problem for future work.

For the present analysis, we can make a very crude estimate of massive string pro- 
duction by using a result on the spectrum of gravitational synchrotron radiation. For a mass in an orbit with period $\omega_{0}$, the power per unit frequency is [14]

$$
\frac{d P}{d \omega} \propto \exp \left(-\frac{\omega}{\omega_{\text {crit }}}\right)
$$

where $\omega_{\text {crit }}=\frac{6}{\pi} \gamma^{2} \omega_{0}$. Thus, for $\omega_{\text {crit }} \ll l_{s}^{-1}$, massive closed strings should play a negligible role, but when gravitons of frequency $l_{s}^{-1}$ are being produced, it is natural to expect massive modes as well. We therefore expect some emission of massive closed strings in processes where $\gamma^{2} \gg \frac{b}{l_{s}}$. This will further increase the rate of energy loss from the revolving brane pair, speeding the trapping and increasing the effective inelasticity of the collision.

\subsection{Summary of the Argument}

For clarity, we will now briefly review our argument that the trapping of relativistic D-branes is powerful and abrupt.

The annulus partition function for open strings between moving D-branes indicates that the density of produced open strings is given, in the relativistic limit, by (24). The characteristic impact parameter below which the backreaction of these strings is strong can then be seen to be $b_{\text {crit }} \sim \eta l_{s}$. This is also the size $r_{\text {nad }}$ of the region in which the open string masses change nonadiabatically.

If the D-branes are assumed to separate to a distance larger than of order $b_{\text {crit }}$, they have left the region of nonadiabaticity, so that (24) applies. The energy (37) in open strings then exceeds the initial brane energy, so that the assumption of significant separation was inconsistent.

The same argument applies when closed string radiation is taken into account. A straightforward estimate of the energy lost to radiation over a distance $b_{\text {crit }}$ shows that the energy transferred to open strings is still sufficient to stop the branes before their separation exceeds $b_{\text {crit }}$.

We expect that a detailed computation of the string production along a decelerating trajectory would show that the stopping length is at most of order $b$, which can be much smaller than $b_{\text {crit }}$. In particular, we expect that in a head-on collision with negligible impact parameter the stopping length would be of order the string length. However, estimates involving (24) are strictly valid only when the branes eventually leave the window of nonadiabaticity, leading to the very conservative estimate $r_{*} \sim b_{\text {crit }}=\eta l_{s}$.

A few potential objections remain. First of all, one might worry that the branes somehow slow down before reaching each other, so that at the moment of closest approach the velocities are nonrelativistic. In this case excited open strings would not be produced and we would simply have field theory trapping. We have already 
explained in $\S 4.3$ that if the branes slow down exclusively due to open string production, then they will still experience rapid trapping. Then, we showed in $§ 5.1$ that additional loss of energy through closed string radiation also does not ruin the trapping.

A final worry is that the branes could interact by creating string pairs at extremely high excitation levels. A vanishingly small number density of arbitrarily highly excited strings (with level much higher than $\eta^{2}$ ) could absorb all the initial kinetic energy and yet not generate a strong attractive force between the branes. However, we have seen that in fact string production peaks around level $n_{\text {peak }} \approx \eta^{2}\left(2 \pi^{2}\right)^{-1}$, which is sufficiently small to ensure that the trapping is strong.

We therefore conclude that D-branes in relativistic motion generically trap each other through copious production of open strings, with a trapping length no larger than the size $\eta l_{s}$ of the nonadiabatic region. A sizeable fraction of the initial energy is eventually emitted in the form of massless closed string radiation.

The limitations to our argument which we have discussed above make it challenging to precisely and controllably compute the stopping length in an ultrarelativistic Dbrane collision. However, these issues, and others - such as massless and massive closed string radiation, annihilation of the produced strings, and dilution of the produced strings in a cosmological background - do not in any way weaken our argument that the brane collision is inelastic. In fact, it is easy to see that radiation, annihilation, and dilution all extract energy from the brane system, slowing the brane motion. (See [1] for an analysis of these issues in the nonrelativistic context.) Happily, for applications to cosmological models, it is the inelasticity rather than the stopping length which is most immediately relevant.

\subsection{Regime of Validity and Control}

We will now examine the characteristics of the trapping process as a function of the dimensionless parameters $g_{s}, b, \eta$.

First of all, we will never work at strong string coupling $\left(g_{s}>1\right)$, since then we would have to include higher string loop effects. Furthermore, at strong coupling the D-branes become very light, and their Compton wavelength $\lambda_{D}$ grows. We require $\lambda_{D} \ll b$ so that we can neglect these quantum effects.

Secondly, we should require that the Schwarzschild radius $R_{s}$ of the D-brane is negligibly small compared to $b$. To estimate this, we treat the $D p$-brane as a point source in $10-p$ dimensions. The black hole solution in $(10-p)$ dimensions for a p-dimensional extended object of tension $\mathrm{T}$ and zero charge is [18]

$$
T=\left(\frac{8-p}{7-p}\right) \frac{R_{s}^{7-p}}{(2 \pi)^{7} d_{p} g_{s}^{2}}
$$


where $d_{p}=2^{5-p} \pi^{\frac{5-p}{2}} \Gamma\left(\frac{7-p}{2}\right)$.

We are interested in the limit of zero charge because the highly-boosted branes have far greater effective mass than the BPS bound requires. Note that in fact the metric for one of these moving branes is of a shock-wave form, not a static black hole. We are imagining that the branes collide inelastically and then asking whether the Schwarzschild radius of the excited remnant, seen in the center of mass frame, is comparable to the initial impact parameter.

In this scheme, the effective tension is the center-of-mass energy $2 T_{p} \gamma \approx T_{p} e^{\eta / 2}$. We therefore find, using the tension of a $p$-brane,

$$
\left(\frac{R_{s}}{l_{s}}\right)^{7-p}=g_{s}\left(\frac{7-p}{8-p}\right)(2 \pi)^{7-p} d_{p} e^{\eta / 2}
$$

from which we conclude that for $p<7$, the Schwarzschild radius can be made parametrically less than any given impact parameter by reducing the string coupling.

Let us now fix $b$ and $\eta$ and take the string coupling to be small enough so that string loops, the brane Schwarzschild radius, and the brane Compton wavelength can be neglected. As we further decrease the coupling, the brane becomes heavier and the stopping length becomes greater. Now, recall that when we examined the open string production along a constant-velocity path, we found an energetic inconsistency: unless the coupling was exponentially small, the open string energy exceeded the initial kinetic energy of the system. Of course, deceleration reduces string production, so for any controllable coupling the energy in open strings will not exceed the initial energy. However, we can still define a value of $g_{s}$ at which the energetics is consistent even before we incorporate the deceleration which arises from backreaction. Comparing (48) and (49), we find that the energetics are automatically consistent provided that

$$
g_{s}<2^{3 / 2} \pi^{p / 2-3} \eta^{4-p / 2} e^{-\eta / 2}
$$

Thus, only for exponentially small string coupling are the branes so heavy that they stretch the open strings substantially before coming to rest.

\section{Discussion}

We have argued that the relativistic scattering of Dp-branes, $p>0$, at small impact parameters is almost completely inelastic as a result of pair production of excited open strings. The time-dependence induces production of an extremely high density of highly-excited, stretched open strings, which rapidly draw the branes into a tight

orbit whose radius is of order the impact parameter. The resulting acceleration results in significant closed string radiation, which acts to further brake the motion. 
Powerful stringy trapping of this sort occurs whenever the impact parameter, measured in string units, is small compared to the rapidity $\eta$. This is a much larger range of distances than that controlled by collision of the stringy halos of the two branes, whose radius grows as $\sqrt{\eta}$. Moreover, the strength of this stringy trapping was a surprise: it does not follow from summing the low-velocity result of 1 over the string spectrum. Instead, the velocity-dependence of stretched string masses enters in a crucial way to enhance the production effect.

Our result, which is essentially a simple observation about the quantum-corrected dynamics of D-branes, has obvious implications for scenarios involving branes in relativistic motion. One example ${ }^{1}$ is the stage of reheating in cosmological models with fast-moving branes and antibranes. Brane-antibrane inflation models typically end with the condensation of the open string tachyon, leaving a dust of closed strings in the bulk as well as excited open strings on any remaining branes [19. Despite much effort, this process is not fully understood [20]. Suppose, however, that the antibrane is moving relativistically toward the end of its evolution, and then passes by or collides with a stack of branes. (Ultrarelativistic brane motion is natural in the DBI models [21, 22], for example, and could occur elsewhere.) In this case tachyon condensation governs only a small fraction of the energy released; most of the kinetic energy goes into open string pair production. Thus, reheating in such a model proceeds by stringy trapping.

More speculatively, moduli trapping may be a useful mechanism for vacuum selection [1], as it gives a dynamical explanation for the presence of enhanced symmetry. (See also [23, 24] for related work on moduli dynamics in string/M theory.) The stringy trapping presented here extends the trapping proposal not just to a new parameter range, but to a regime where the strength of the effect increases dramatically.

The inelasticity of D-brane scattering may be viewed as a calculable example of a more general question: to what extent do particle, string, and brane production affect motion toward or away from a given 'singular' configuration? Time-dependent orbifolds [25, 26, 27, 28, 29, 30, 31] (see also [32] and references therein) provide a relatively tractable setting for such a question. Berkooz and Pioline [30] and Berkooz, Pioline and Rozali [31] have emphasized the possibility of resolving a spacelike singularity through the pair production and condensation of winding strings. It would be very interesting to extend these results and repair more general spacelike singularities through the production of branes or strings; see [33] for work in this direction. Our analysis suggests that string production could be surprisingly important in such a setting.

Another interesting open question is whether the inelasticity of quantum-corrected D-brane collisions can be used to place bounds on the elasticity of other sorts of col-

\footnotetext{
${ }^{1}$ We are grateful to S. Kachru for suggesting this.
} 
lisions. In the cyclic universe model [34, 35], the orbifold boundaries of heterotic M-theory [36] approach each other and collide. An intrinsic assumption of these cyclic models is that the collision is very nearly elastic; this is essential to make possible a large number of collisions and the associated cyclic behavior. Our result makes it plain that D-brane collisions, which appear elastic classically, are highly inelastic when the quantum effects associated to fundamental strings are included.

In the cyclic model, the M2-branes stretched between the boundaries become tensionless at the instant of collision. In the weakly-coupled four-dimensional description these objects are heterotic strings whose tension, in four-dimensional Planck units, goes to zero at the moment of impact. Because the masses vary rapidly during the collision, the nonadiabaticity is large and we expect copious production of these strings. It would be extremely interesting to compute the energy loss through this string/membrane production and to understand the implications for the cyclic models 37.

We should point out that in the most realistic cyclic models, the brane velocities are required, for phenomenological reasons, to be nonrelativistic. ${ }^{2}$ The results in this paper appear to give an independent upper bound on the velocity of the branes before collision - this bound is one which is required for the self-consistency of the model, rather than one imposed by observational requirements. However, this argument is qualitative at present; an explicit extension of our results involving stretched fundamental strings to the case of stretched membranes would be nontrivial.

\section{Acknowledgements}

We are very grateful to E. Silverstein for collaboration in the early stages of this project and for helpful comments throughout. We would also like to thank R. Bousso, M. Fabinger, B. Freivogel, O. Ganor, S. Giddings, S. Gubser, P. Horava, S. Kachru, X. Liu, A. Maloney, M. M. Sheikh-Jabbari, S. Shenker, and P. J. Steinhardt for helpful discussions, and S. Gubser, E. Silverstein, and P. J. Steinhardt for comments on the manuscript. L.M. was supported in part by the Department of Energy under contract number DE-AC03-76SF00515, and is grateful to the organizers of the 2004 PiTP program in String Theory, where a portion of this work was done. I.M. was supported in part by the Director, Office of Science, Office of High Energy and Nuclear Physics, of the U.S. Department of Energy under Contract DE-AC03-76SF00098, and in part by the NSF under grant PHY-0098840 and grant PHY-0244900. I.M. also wishes to thank the Aspen Center for Physics for hospitality during the final stages of this work.

\footnotetext{
${ }^{2}$ We are grateful to P. J. Steinhardt for helpful discussions on this point.
} 


\section{Appendix}

In this section we collect various identities about the elliptic theta functions. Because of the existence of several canonical notations for these functions, we define the functions as used in the paper.

The theta functions are often expressed in terms of the variables $\nu$ and $\tau$, or in terms of the nome $q=\exp (2 \pi i \tau)$ and $z=\exp (2 \pi i \nu)$. The four theta functions are written down below in both their series and product forms:

$$
\begin{aligned}
\theta_{00}(\nu, \tau)=\theta_{3}(\nu \mid \tau) & =\sum_{n=-\infty}^{n=\infty} q^{n^{2} / 2} z^{n}=\prod_{m=1}^{\infty}\left(1-q^{m}\right)\left(1+z q^{m-1 / 2}\right)\left(1+z^{-1} q^{m-1 / 2}\right) \\
\theta_{01}(\nu, \tau)=\theta_{4}(\nu \mid \tau) & =\sum_{n=-\infty}^{n=\infty}(-1)^{n} q^{n^{2} / 2} z^{n}=\prod_{m=1}^{\infty}\left(1-q^{m}\right)\left(1-z q^{m-1 / 2}\right)\left(1-z^{-1} q^{m-1 / 2}\right) \\
\theta_{10}(\nu, \tau)=\theta_{2}(\nu \mid \tau) & =\sum_{n=-\infty}^{n=\infty} q^{(n-1 / 2)^{2} / 2} z^{n-1 / 2} \\
& =2 e^{\pi i \tau / 4} \cos (\pi \nu) \prod_{m=1}^{\infty}\left(1-q^{m}\right)\left(1+z q^{m}\right)\left(1+z^{-1} q^{m}\right) \\
-\theta_{11}(\nu, \tau)=\theta_{1}(\nu \mid \tau) & =i \sum_{n=-\infty}^{n=\infty}(-1)^{n} q^{(n-1 / 2)^{2} / 2} z^{n-1 / 2} \\
& =2 e^{\pi i \tau / 4} \sin (\pi \nu) \prod_{m=1}^{\infty}\left(1-q^{m}\right)\left(1-z q^{m}\right)\left(1-z^{-1} q^{m}\right) .
\end{aligned}
$$

In addition to the theta functions, we shall also need the Dedekind eta function:

$$
\eta(\tau)=q^{1 / 24} \prod_{m=1}^{\infty}\left(1-q^{m}\right)=\left[\frac{\partial_{\nu} \theta_{11}(0, \tau)}{-2 \pi}\right]^{1 / 3} .
$$

These functions have the following modular transformation properties:

$$
\begin{aligned}
& \theta_{00}(\nu / \tau,-1 / \tau)=(-i \tau)^{1 / 2} \exp \left(\pi i \nu^{2} / \tau\right) \theta_{00}(\nu, \tau) \\
& \theta_{01}(\nu / \tau,-1 / \tau)=(-i \tau)^{1 / 2} \exp \left(\pi i \nu^{2} / \tau\right) \theta_{10}(\nu, \tau) \\
& \theta_{10}(\nu / \tau,-1 / \tau)=(-i \tau)^{1 / 2} \exp \left(\pi i \nu^{2} / \tau\right) \theta_{01}(\nu, \tau) \\
& \theta_{11}(\nu / \tau,-1 / \tau)=-(-i \tau)^{1 / 2} \exp \left(\pi i \nu^{2} / \tau\right) \theta_{11}(\nu, \tau) \\
& \eta(-1 / \tau)=(-i \tau)^{1 / 2} \eta(\tau) .
\end{aligned}
$$

We will often need the asymptotic behavior of the theta and eta functions. When $q \ll 1$ we can immediately find the asymptotics using the above expansions, whereas for $q \rightarrow 1$ we must first perform a modular transformation. 
The asymptotic behavior of a particular combination will be especially helpful. Define the fermionic partition function $Z(\tau) \equiv \frac{1}{2} \theta_{10}^{4}(0 \mid \tau) \eta(\tau)^{-12}$. Then for $-i \tau \equiv s \gg 1$ we have

$$
Z(i s)=8+\mathcal{O}\left(e^{-2 \pi s}\right)
$$

whereas for $s \ll 1$ we find, using the modular transformations above,

$$
Z(i s)=\frac{1}{2} s^{4} \exp \left(\frac{\pi}{s}\right)\left(1+\mathcal{O}\left(e^{-\frac{\pi}{s}}\right)\right)
$$

We will also need a few identities involving the theta functions:

$$
\begin{aligned}
& \theta_{00}^{4}(0, \tau)-\theta_{01}^{4}(0, \tau)-\theta_{10}^{4}(0, \tau)=0 \quad \theta_{11}(0, \tau)=0 \\
& \prod_{a=1}^{4} Z_{0}^{0}\left(\phi_{a}, i t\right)-\prod_{a=1}^{4} Z_{1}^{0}\left(\phi_{a}, i t\right)-\prod_{a=1}^{4} Z_{0}^{1}\left(\phi_{a}, i t\right)-\prod_{a=1}^{4} Z_{1}^{1}\left(\phi_{a}, i t\right)=2 \prod_{a=1}^{4} Z_{1}^{1}\left(\phi_{a}^{\prime}, i t\right),
\end{aligned}
$$

where

$$
\begin{array}{ll}
Z_{\beta}^{\alpha}(\phi, i t)=\frac{\theta_{\alpha \beta}(i \phi t / \pi, i t)}{\exp \left(\phi^{2} t / \pi\right) \eta(i t)} & \\
\phi_{1}^{\prime}=\frac{1}{2}\left(\phi_{1}+\phi_{2}+\phi_{3}+\phi_{4}\right) & \phi_{2}^{\prime}=\frac{1}{2}\left(\phi_{1}+\phi_{2}-\phi_{3}-\phi_{4}\right) \\
\phi_{3}^{\prime}=\frac{1}{2}\left(\phi_{1}-\phi_{2}+\phi_{3}-\phi_{4}\right) & \phi_{4}^{\prime}=\frac{1}{2}\left(\phi_{1}-\phi_{2}-\phi_{3}+\phi_{4}\right) .
\end{array}
$$

The identity (75) leads in the case $\phi_{2}=\phi_{3}=\phi_{4}=0$ to

$$
2 \theta_{11}^{4}(\nu / 2, \tau)=\theta_{00}(\nu, \tau) \theta_{00}^{3}(0, \tau)-\theta_{01}(\nu, \tau) \theta_{01}^{3}(0, \tau)-\theta_{10}(\nu, \tau) \theta_{10}^{3}(0, \tau) .
$$

\section{References}

[1] L. Kofman et. al., "Beauty is attractive: Moduli trapping at enhanced symmetry points," hep-th/0403001.

[2] J. S. Schwinger, "On gauge invariance and vacuum polarization," Phys. Rev. 82 (1951) 664-679.

[3] C. Bachas and M. Porrati, "Pair creation of open strings in an electric field," Phys. Lett. B296 (1992) 77-84, hep-th/9209032.

[4] C. Bachas, "D-brane dynamics," Phys. Lett. B374 (1996) 37-42, hep-th/9511043.

[5] M. R. Douglas, D. Kabat, P. Pouliot, and S. H. Shenker, "D-branes and short distances in string theory," Nucl. Phys. B485 (1997) 85-127, hep-th/9608024 
[6] L. Kofman, A. D. Linde, and A. A. Starobinsky, "Towards the theory of reheating after inflation," Phys. Rev. D56 (1997) 3258-3295, hep-ph/9704452.

[7] G. N. Felder, L. Kofman, and A. D. Linde, "Inflation and preheating in NO models," Phys. Rev. D60 (1999) 103505, hep-ph/9903350.

[8] J. Polchinski, "String theory. Vol. 2: Superstring theory and beyond," Cambridge, UK: Univ. Pr. (1998) 531 pp.

[9] J. Polchinski, "String theory. Vol. 1: An introduction to the bosonic string," Cambridge, UK: Univ. Pr. (1998) 402 pp.

[10] C. P. Bachas, "Lectures on D-branes," hep-th/9806199.

[11] D. J. H. Chung, "Classical inflaton field induced creation of superheavy dark matter," Phys. Rev. D67 (2003) 083514, hep-ph/9809489.

[12] S. S. Gubser, "String production at the level of effective field theory," Phys. Rev. D69 (2004) 123507, hep-th/0305099.

[13] S. S. Gubser, "String creation and cosmology," hep-th/0312321.

[14] R. Breuer, "Gravitational perturbation theory and synchrotron radiation," Berlin: Springer-Verlag (1975) 196 pp.

[15] P. C. Peters, "Relativistic gravitational bremsstrahlung," Phys. Rev. D1 (1970) $1559-1571$.

[16] M. Abou-Zeid and M. S. Costa, "Radiation from accelerated branes," Phys. Rev. D61 (2000) 106007, hep-th/9909148.

[17] J. D. Jackson, "Classical Electrodynamics," New York: Wiley (1999) 808 pp.

[18] G. T. Horowitz and A. Strominger, "Black strings and P-branes," Nucl. Phys. B360 (1991) 197-209.

[19] A. Sen, "Tachyon condensation on the brane antibrane system," JHEP 08 (1998) 012, hep-th/9805170

[20] N. Lambert, H. Liu, and J. Maldacena, "Closed strings from decaying D-branes," hep-th/0303139.

[21] E. Silverstein and D. Tong, "Scalar speed limits and cosmology: Acceleration from D- cceleration," hep-th/0310221.

[22] M. Alishahiha, E. Silverstein, and D. Tong, "DBI in the sky," hep-th/0404084. 
[23] S. Watson, "Moduli stabilization with the string Higgs effect," hep-th/0404177.

[24] L. Jarv, T. Mohaupt, and F. Saueressig, "M-theory cosmologies from singular Calabi-Yau compactifications," JCAP 0402 (2004) 012, hep-th/0310174.

[25] H. Liu, G. Moore, and N. Seiberg, "Strings in a time-dependent orbifold," JHEP 06 (2002) 045, hep-th/0204168

[26] H. Liu, G. Moore, and N. Seiberg, "Strings in time-dependent orbifolds," JHEP 10 (2002) 031, hep-th/0206182

[27] G. T. Horowitz and J. Polchinski, "Instability of spacelike and null orbifold singularities," Phys. Rev. D66 (2002) 103512, hep-th/0206228.

[28] M. Fabinger and J. McGreevy, "On smooth time-dependent orbifolds and null singularities," JHEP 06 (2003) 042, hep-th/0206196.

[29] M. Fabinger and S. Hellerman, "Stringy resolutions of null singularities," hep-th/0212223.

[30] B. Pioline and M. Berkooz, "Strings in an electric field, and the Milne universe," JCAP 0311 (2003) 007, hep-th/0307280.

[31] M. Berkooz, B. Pioline, and M. Rozali, "Closed strings in Misner space: Cosmological production of winding strings," hep-th/0405126.

[32] L. Cornalba and M. S. Costa, "Time-dependent orbifolds and string cosmology," Fortsch. Phys. 52 (2004) 145-199, hep-th/0310099.

[33] J. J. Friess, S. S. Gubser, and I. Mitra, "String creation in cosmologies with a varying dilaton," Nucl. Phys. B689 (2004) 243-256, hep-th/0402156.

[34] J. Khoury, B. A. Ovrut, N. Seiberg, P. J. Steinhardt, and N. Turok, "From big crunch to big bang," Phys. Rev. D65 (2002) 086007, hep-th/0108187.

[35] P. J. Steinhardt and N. Turok, "A cyclic model of the universe," hep-th/0111030.

[36] P. Horava and E. Witten, "Eleven-Dimensional Supergravity on a Manifold with Boundary," Nucl. Phys. B475 (1996) 94-114, hep-th/9603142.

[37] M. Perry, P. J. Steinhardt, and N. Turok, work in progress. 